Published in: Photomedicine and Laser Surgery. December 2009, 27(6): 929935.

\title{
Analysis of the Systemic Effect of Red and Infrared Laser Therapy on Wound Repair
}

\author{
Simone Marja Rodrigo, ${ }^{1}$ \\ Alexandre Cunha, ${ }^{2}$ \\ Daniel Humberto Pozza, ${ }^{3}$ \\ Diego Segatto Blaya, ${ }^{4}$ \\ João Feliz Moraes, ${ }^{5}$ \\ João Batista Blessmann Weber, ${ }^{1}$ and \\ Marília Gerhardt de Oliveira ${ }^{1}$ \\ ${ }^{1}$ School of Dentistry, Pontifícia Universidade Católica do Rio Grande do Sul, \\ Porto Alegre, Brazil. \\ ${ }^{2}$ School of Physics, Pontifícia Universidade Católica do Rio Grande do Sul, \\ Porto Alegre, Brazil. \\ ${ }^{3}$ Instituto de Histologia e Embriologia, Faculdade de Medicina, Universidade do \\ Porto, Portugal. \\ ${ }^{4}$ School of Dentistry, Universidade Federal de Santa Maria, Santa Maria, Brazil. \\ ${ }^{5}$ School of Mathematics, Pontifícia Universidade Católica do Rio Grande do Sul, \\ Porto Alegre, Brazil.
}

\section{Abstract}

Objective: To evaluate, using histological analysis, the systemic action and repair process of wounds produced on the back of rats and treated with red, infrared, or both lasers applied directly or indirectly to the wounds. Background Data: Skin tissue repair and wound healing are complex processes that involve a series of dynamic events. Many benefits are associated with biomodulation uisng laser therapy. Methods: Thirty-six male Wistar rats were divided into four groups: control (without laser), red laser (aluminium gallium indium phosphide (AIGalnP); $\lambda=685 \mathrm{~nm} ; \lambda=0.0314 \mathrm{~cm}^{2} ; C W ; P=30 \mathrm{~mW} ; \mathrm{D}=20 \mathrm{~J}$, time of irradiation $=667 \mathrm{sec}$ ), infrared laser (gallium-aluminum-arsenide (GaAlAs): $\lambda=$ $830 \mathrm{~nm} ; \lambda=0.0314 \mathrm{~cm}^{2} ; \mathrm{CW} ; \mathrm{P}=50 \mathrm{~mW} ; \mathrm{D}=20 \mathrm{~J}$, time of irradiation $=401 \mathrm{sec}$ ), and both lasers (infrared laser: GaAlAs; $\lambda=830 \mathrm{~nm} ; \lambda=0.0314 \mathrm{~cm}^{2}$; CW; $P=50$ $\mathrm{mW}$; $\mathrm{D}=10 \mathrm{~J}$, time of irradiation $=201 \mathrm{sec}+$ red laser: AlGalnP; $\lambda=685 \mathrm{~nm} ; \lambda=$ $0.0314 \mathrm{~cm}^{2} ; \mathrm{CW} ; \mathrm{P}=30 \mathrm{~mW} ; \mathrm{D}=10 \mathrm{~J}$, time of irradiation $=334 \mathrm{sec}$; total dose $=$ $20 \mathrm{~J}$ ). Three subgroups were formed according to observation time points. Three wounds were produced on the back of each animal. Only the wound closest to the head was irradiated in the experimental groups. For the evaluation of skin reaction and wound healing, three animals of each group were killed at 3,5 , and 7 days postoperatively. The irradiation protocol established 48-hour intervals between applications, with the first application immediately after the surgical procedure. Results: In the red and infrared laser group, healing was more advanced in the wound located furthest from the point of laser application. The most effective healing of a proximal wound was verified 
in the control group on the 7th postoperative day. Conclusion: The combined application of red and infrared lasers resulted in the most evident systemic effect on the repair of skin wounds produced in rats.

\section{Introduction}

SKIN TISSUE REPAIR AND WOUND HEALING are complex processes that occur in sequential phases: coagulation, inflammation, granulation, wound contraction, and tissue remodeling. The introduction of laser therapy opened a new perspective in wound management because of its clinical efficacy in tissue healing. The basic principle of laser therapy is that monochromatic light has a wavelength that is capable of affecting cell behavior without significant heating effects.

Many benefits, including stimulation or inhibition of physiological, biochemical, or proliferative activities, are associated with biomodulation using laser therapy. Beneficial effects demand a threshold of radiation exposure, and the variation of the laser fluency is calculated according to the desired effect. ${ }^{-}$The effects are dose-dependent, and high energies, within specific limits for each tissue-laser combination, may be harmful. Because of the diffusion of laser light upon penetrating tissue, a short length of the appropriate radiation may be sufficient to achieve the biomodulation process. Local irradiation may produce systemic effects, but the direct irradiation of tissue may not always be necessary because irradiation penetrates deeply and produces distant effects.

Al-Watban and Zhang ${ }^{2}$ conducted a study to define the ideal dose of laser light in the treatment of skin wounds on the backs of rats. In the comparison of fluency of 10,20 , and $30 \mathrm{~J} / \mathrm{cm}^{2}$ applied three times weekly, the best results were obtained with $20 \mathrm{~J} / \mathrm{cm}^{2}$. In ascending order, the best results for skin wounds were verified to helium-neon (HeNe; 632.8 nm), gallium-aluminum-arsenide (GaAlAs; $780 \mathrm{~nm})$, argon $(488-514 \mathrm{~nm})$, GaAIAs $(830 \mathrm{~nm})$, helium-cadmium $(442 \mathrm{~nm})$, and krypton $(640-670 \mathrm{~nm})$. Their findings confirm that the depth of laser penetration, which is associated with the wavelength used, is not proportional to the biomodulation effects of laser therapy.

Based on these outcomes reported by Al-Watban and Zhang, ${ }^{\underline{2}}$ other authors used a fluency of $20 \mathrm{~J} / \mathrm{cm}^{2}$ to evaluate the healing of skin wounds on the back of rats. ${ }^{3,4}$ Their analyses revealed that the assembly between wavelengths of 685 $\mathrm{nm}$ and $830 \mathrm{~nm}\left(10 \mathrm{~J} / \mathrm{cm}^{2}\right.$ each; total $\left.=20 \mathrm{~J} / \mathrm{cm}^{2}\right)$ promoted the best skin wound healing. $\underline{3}$

In a study to evaluate the systemic effects of low-level laser therapy (LLLT), rats with injuries in the peripheral and central nervous system, skin wounds, and burns were irradiated with HeNe laser $(632.8 \mathrm{~nm})$ at a power of $16 \mathrm{~mW}$ and different energy densities for 21 days. $\frac{5}{}$ Laser was applied only to the right side of the animals after bilateral skin wounds were produced. The authors observed better healing of both wounds than in the control group, which did not receive irradiation. Statistical analysis showed a significant difference between the 
group treated with laser and the control group. The authors concluded that HeNe laser had a marked systemic effect on the skin and adjacent tissues, as well as on the severely damaged peripheral nerves and the corresponding regions of the marrow. According to the authors, the effects persisted for a long time after laser irradiation. Those authors suggested that systemic effects are relevant for the clinical application of laser therapy and for basic research about its possible mechanisms of action.

Another study evaluated the systemic effects of laser irradiation on healing of wounds produced on the skin of rats. ${ }^{\underline{6}}$ Two parallel incisions were made on the back of each animal and immediately sutured. After surgery, one wound of each rat was irradiated with LLLT at wavelength $670 \mathrm{~nm}$ and a daily fluency of 30 $\mathrm{J} / \mathrm{cm}^{2}$. The second wound did not receive irradiation and was adopted as a control group. The wounds were evaluated at 24, 48, 72, 96, 120, 144, and 168 h postoperatively. The comparison with nonirradiated wounds showed that laser stimulation accelerated the inflammatory phase, as well as the proliferative and maturation phases. The regeneration of damaged epidermis and the repair of striated muscle were also positively stimulated. However, other researchers did not obtain different results in the comparison of irradiated and nonirradiated wounds of the same animal. $\frac{7-9}{2}$ The fact that they used a contralateral wound as control and that the same animal served as experimental and control subject may explain their findings. Their results support the idea that lasers do not act only on the sites of irradiation but also produce systemic effects.

The aim of this study was to evaluate the systemic action of LLLT and repair process of skin wounds in rats with the application of LLLT at wavelengths of $830 \mathrm{~nm}$ and $685 \mathrm{~nm}$, separately or in association.

\section{Materials and Methods}

The Research Ethics Committee of the School of Dentistry, Pontifícia Universidade Católica do Rio Grande do Sul, Porto Alegre, Brazil, evaluated and approved this study under approval number 0032/5. This research was conducted in accordance with the ethical principles of animal experimentation and the Brazilian norms for the practical, educational, and scientific uses of vivisection established in Law no. 6.638/79. ${ }^{-}$

Thirty-six male Wistar rats, clinically healthy and weighing between 200 and 250 $\mathrm{g}$ were randomly selected, weighed, and divided into four groups [I, II, III, IV] of nine animals each. ${ }^{-}$Each group was then divided into three subgroups $(A, B$, and $C$ ) according to each observation healing time. All groups of animals were examined at 3,5 , and 7 days postoperatively. During the study, the animals were kept in plastic cages, with a metal top and pine sawdust as bedding, in normal laboratory conditions (room temperature $22^{\circ} \mathrm{C}, 12-\mathrm{h}$ light/dark cycle) and were fed a solid diet, except in the first $24 \mathrm{~h}$ postoperatively, and water ad libitum. 


\section{Surgical procedure}

All the animals received general anesthesia with $20.0 \mathrm{mg} / \mathrm{kg}$ intramusclar Zoletil 50 (Virbac do Brasil, São Paulo, SP, Brazil). After anesthesia, the backs of the animals were shaved, and the regions were cleaned with $2 \%$ chlorhexidine.

A punch was positioned perpendicularly to the surface of the skin on the shaved region, next to the midline, and close to the head of the animal. Circular movements were made to facilitate instrument penetration to a depth of $1 \mathrm{~mm}$. The wounds measured $1 \mathrm{~cm}$ in diameter, which corresponded to the diameter of the instrument used. Soft tissue was carefully removed to obtain a 1-mm-deep wound. The same procedures were followed to produce another wound $7 \mathrm{~cm}$ from the first one and a third wound $7 \mathrm{~cm}$ from the second one. The wounds and the irradiation process are shown in Figure 1.
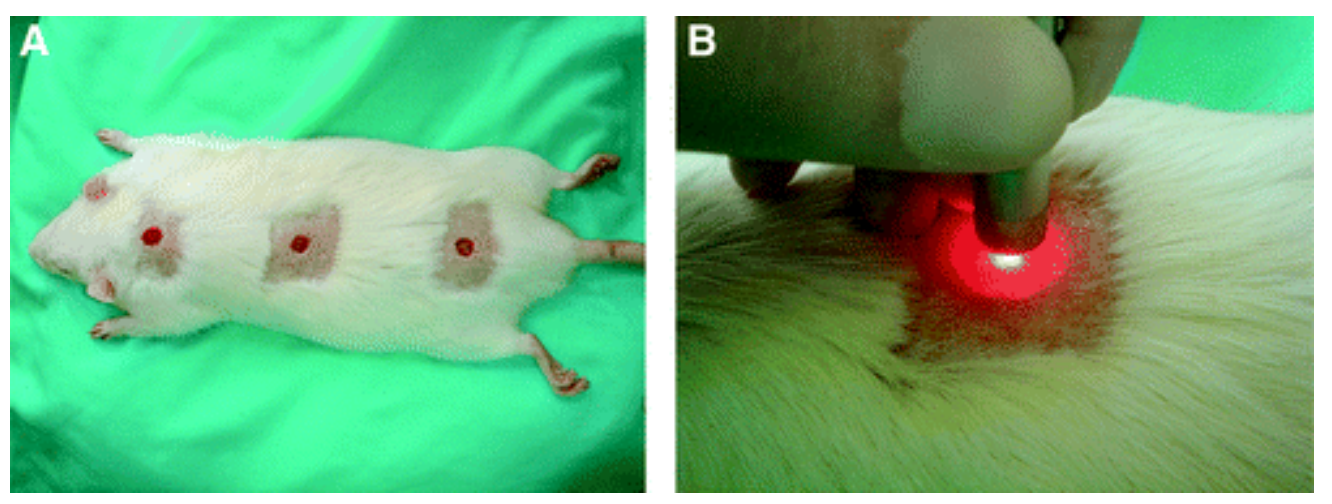

FIG. 1. Experimental procedures. (A) Wounds created with a 1-mm depth and $1-\mathrm{cm}$ diameter. The wounds were made by circular movements using a punch. (B) Laser irradiation using red or infrared or a combination of the two.

The animals were killed at 3,5 , and 7 days postoperatively using a lethal injection $(3-4 \mathrm{~mL})$ of $10 \%$ chloral hydrate. The three wounds of each animal were dissected individually and labeled. After dissection, hematoxylin-eosin $(\mathrm{HE})$ was applied to the wounds for evaluation.

The histological samples were processed at Universidade Federal da Bahia, Brazil, and then submitted to a descriptive and comparative microscopic analysis under light microscopy; images were captured at 100× magnification to analyze the tissue repair process in the biological models. Presence of mononucleated cells was considered characteristic of the chronic inflammatory stage. Collagen synthesis and epithelization were established as the criteria for the occurrence of tissue repair in the biological models. Samples were stained with $\mathrm{HE}$ and analyzed microscopically to evaluate the inflammatory infiltrate, epithelization, and collagen synthesis. The following aspects were evaluated via histological analysis: epithelization, edema, hyperemia, hemorrhage, fibroblasts, lymphocytes, neutrophils, necrosis, foreign body, and isolated connective tissue. Specimens were assigned scores according to healing stage, receiving a 
score of 0 for initial healing stage and 1 for advanced stage. The sum of these scores in the analysis of each wound resulted in a variable called rank of healing.

\section{Statistical analysis}

Statistical analyses were conducted according to the rank of healing of each wound. The Kolmogorov-Smirnov test and the Kruskal-Wallis nonparametric test were used to compare more than two independent groups. A model for mean-comparison analysis was used to analyze data regarding the following effects: Time effect: comparison between time points at 3, 5, and 7 days; Experimental effect: comparison between experimental and control groups; Wound site effect: comparison between wound sites; and Combination effect: comparison between time points, groups, and sites.

The Kolmogorov-Smirnov test was used to evaluate the normality of number variables. Because all variables were nonnormal, the Kruskal-Wallis parametric test was used to compare groups and results at the different time points. SPSS for Windows (SPSS, Inc., Chicago, IL) was used for data analysis.

\section{Laser irradiation}

Group I was the control group and received not irradiation.

The infrared laser used in group II was gallium-aluminum-arsenide (GaAlAs, wavelength $830 \mathrm{~nm}, \lambda=0.0314 \mathrm{~mm}^{2}$, continuous-wave mode, in contact mode, $P$ $=50 \mathrm{~mW})$. The irradiation fluency was $20 \mathrm{~J}$, and the laser was applied continuously to one point immediately after surgery (time of irradiation $=404 \mathrm{sec}$ ) and then every $48 \mathrm{~h}$ until the animal was killed $(3,5$, or 7 days). The total number of applications was two, three, or four.

In group III, continuous wave red laser (aluminium gallium indium phosphide (AIGalnP), wavelength $685 \mathrm{~nm}, \lambda=0.0314 \mathrm{~cm}^{2}$ ), was used for application to one point, in contact mode, $P=30 \mathrm{~mW}$. The fluency was also $20 \mathrm{~J}$ for each application, for a total of two, three, or four applications (time of irradiation $=667$ $\mathrm{sec})$.

In group IV, the two laser types were used in combination. They were applied to one point, in contact mode, in continuous-wave mode at a total fluency of $20 \mathrm{~J}$ for each application (infrared laser: GaAIAs; $\lambda=830 \mathrm{~nm} ; \lambda=0.0314 \mathrm{~cm}^{2} ; \mathrm{CW} ; \mathrm{P}=$ $50 \mathrm{~mW} ; \mathrm{D}=20 \mathrm{~J}$, time of irradiation $=201 \mathrm{sec}+$ red laser: AlGalnP; $\lambda=685 \mathrm{~nm} ; \lambda=$ $0.0314 \mathrm{~cm}^{2} ; \mathrm{CW} ; \mathrm{P}=30 \mathrm{~mW} ; \mathrm{D}=10 \mathrm{~J}$; time of irradiation $=5.34 \mathrm{~min}$ ). First, the infrared laser was used at a fluency of $10 \mathrm{~J} / \mathrm{cm}^{2}$, immediately followed by the red laser application at an equal fluency.

Only the wound closest to the head received irradiation in all test groups (II, III, and IV), and the laser beam was applied to a point in the center of each wound. The relationship between groups and total fluency applied to wounds is shown in Table 1. 


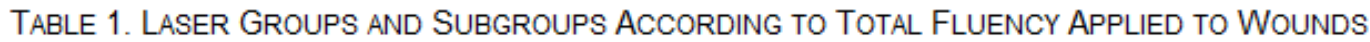

\begin{tabular}{|l|c|c|}
\hline Group & Subgroup & Total fluency (J) \\
\hline II & A & 40 \\
\hline & B & 60 \\
\hline III & C & 80 \\
\hline & A & 40 \\
\hline & B & 60 \\
\hline IV & C & 80 \\
\hline & A & 40 \\
\hline & B & 60 \\
\hline
\end{tabular}

In subgroup A, the laser irradiation was applied after 3 days, in $\mathrm{B}$ after 5 days, and in $\mathrm{C}$ after 7 days.

\section{Results}

The animals remained healthy during the study, and healing of the operated sites was normal, without signs of infection.

Photomicrographs of the HE-stained samples after 3 days of healing (initial healing stage) showed the systemic effect of LLLT on wound repair (Fig. 2).
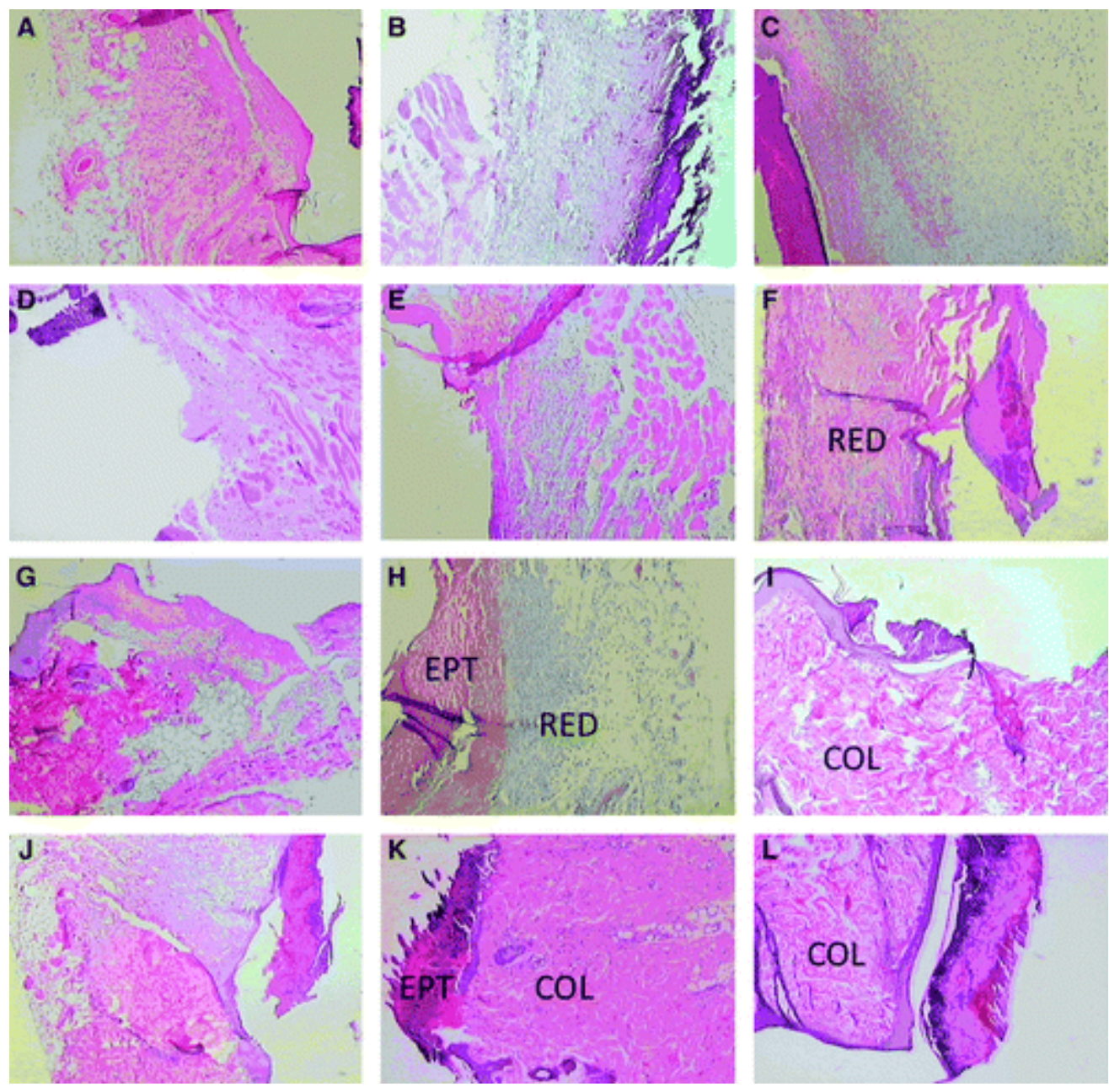
FIG. 2. Photomicrographs of samples (bar $=40 \mathrm{X}$ ) stained with hematoxylineosin after 3 days of healing (initial healing stage) showing the systemic effect of low-level laser therapy on wound repair. All images are distributed in the dorsal, medial, and caudal region from left to right. (A-C) control group (I); (DF) infrared laser group (II); (G-I) red laser group (III); and (J-L) combined infrared and red laser group (IV). Effects on the repair process: reduced local inflammation (RED), organized collagen (COL), and faster epithelization (EPT).

The analysis of the rank of healing according to time points revealed statistically significant differences according to the Kruskal-Wallis test $(p=0.000)$ (Fig. 3). The wounds had the best healing ranks on the 7th day.

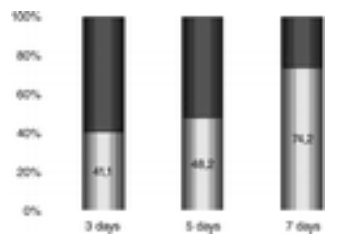

FIG. 3. Rank of scarring according to number of days.

The analysis of rank of healing according to type of laser applied revealed no statistically significant differences in wound healing between groups according to the Kruskal-Wallis test $(p=0.26)$ (Fig. 4). The best mean ranks of healing were found for the groups in which red laser or the combination red and infrared laser was used.

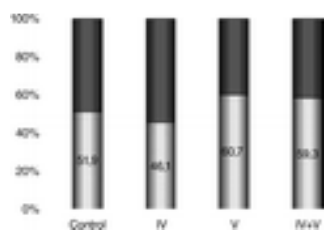

FIG. 4. Rank of scarring according to group.

The analysis of healing rank according to wound site revealed no statistically significant differences in wound healing according to the Kruskal-Wallis test $(p=$ 0.33) (Fig. 5). However, the wounds located in the intermediate region had a better healing rank than those in the other sites. The wounds in the regions that received laser applications (dorsal region) had the worst means.

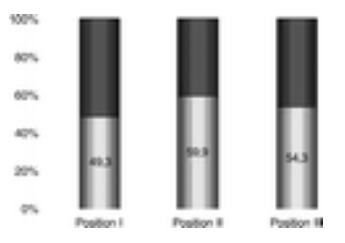

FIG. 5. Rank of scarring according to position. 
The analysis of healing rank according to wound site and time points revealed statistically significant differences in wound healing for the third wound on the 3 rd $(p=0.03)$ and 7 th $(p=0.04)$ days when red and infrared lasers were used in combination (Table 2). Moreover, the most distal wounds had healed better at 3 days postoperatively in group IV, in which red and infrared lasers were used in combination, when compared with the other experimental groups. At 7 days, the rank of healing of the same wounds was higher in the control group, in which no laser was applied.

\section{Discussion}

In agreement with worldwide bioethical paradigms, the number of animals used in this study was limited to three for each subgroup.

The results of several studies are contradictory, partly because of different indications, wide variation of irradiation parameters, and even the difficulty in objectively measuring possible effects of different irradiation densities. Moreover, studies should not only investigate the mechanisms of action and the establishment of criteria for LLLT effects. It is also necessary and important to simplify evaluations and interpretation of the outcomes., $., 10,11$

This study showed that the application of laser directly to the wounds stimulated their healing, as well as healing of the wounds distant from the point of application. This was observed especially in the initial phase of wound repair but at a greater intensity in the group in which red and infrared lasers were used in combination. Therefore, the effects of laser applications are unclear; the biomodulation promoted by laser in cells at the site of application may also occur in tissues located distant from that point. Such stimulation of distant cells may be associated with the increase of the number of mitoses, which may modulate cell physiology in different sites. $\underline{5}$

Wounds located where laser was applied had the worst mean ranks of healing and those in the intermediate position had the best. Although all procedures were performed with utmost care and technical expertise, not causing any extra trauma to the animal, the contact between the tip of the laser device, laser therapy and the tissues and the direct administration of light may explain the results found in our study.

Wounds irradiated with infrared laser had a remarkably lower mean rank of healing than the wounds that received the other two types of therapy under study. The wavelength used, which promotes better tissue penetration and does not act intensively in the superficial layers, such as the epithelium, may explain this result. Red laser was a factor that determined greater mean ranks of healing of irradiated areas, followed by the combined use of red and infrared lasers. $, \underline{2}, \underline{12}$

The areas irradiated with red laser had better mean ranks of healing than the control and infrared laser groups, although this difference was not statistically 
significant. Histological analysis showed a large amount of vascular tissue and blood cells, which may suggest that the laser increased vascularization. Lasers may also enlarge lymphatic vessels, which may promote better oxygenation of tissue under repair and therefore reduce edema. $\underline{10,13}$

In this study, each laser application fluency was $20 \mathrm{~J}$ at $830 \mathrm{~nm}$ or $685 \mathrm{~nm}$. Differences in laser fluencies used for LLLT were found in the literature, which complicated the comparison between studies. $\underline{6}$

In others studies, daily fluencies ranged from 0.2 to $30 \mathrm{~J} / \mathrm{cm}^{2}$. In the study conducted by Al-Watban and Zhang, ${ }^{2}$ ideal fluencies and wavelengths for the treatment of wounds of rats were evaluated, and the best results were achieved with $20 \mathrm{~J} / \mathrm{cm}^{2}$ and 640 to $670 \mathrm{~nm}$.

Healthy persons rarely demand treatment for wound healing. $\frac{14}{1}$ The use of light may have favorable effects on wounds that do not heal. Delays in wound healing usually result from complications such as bad nutrition, diabetes, malignancy, ischemia due to vascular disease, and advanced age. $\frac{15}{}$

Collagen output in wounds of patients with diabetes may be modulated using laser therapy. The process by which laser biostimulation facilitates collagen output in wound healing of patients with diabetes may involve several

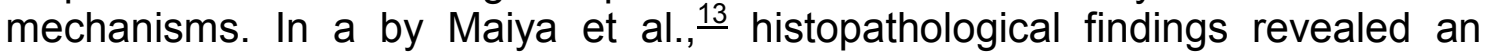
increase in the proliferation of fibroblasts and epithelization. That suggests that the use of laser at $638 \mathrm{~nm}$ may modulate cell proliferation and release factors that promote fibroblast growth. The systemic effect of LLLT also was evaluated in ulcers and gangrene of patients with diabetes in a study by Schindl et al., $\frac{16}{}$ who found significant improvement in local microcirculation.

Because of some limitations of this study, such as wounds located close to each other and the fact that we did not assess systemic blood levels of cytokines, chemokines, and growth factors, new studies should be conducted to evaluate the therapeutic efficacy of LLLT used in the treatment of wounds whose healing is delayed. In addition to their biomodulation effects on the sites of application, lasers seems to affect cell functioning in the whole organism. Therefore, it is important to standardize methods for laser application and for the evaluation of its possible systemic effects.

\section{Conclusion and Summary}

The application of laser at wavelengths of 830 or $685 \mathrm{~nm}$ had systemic effects on the process of repair of standardized skin wounds. A systemic effect of laser was found in the wounds located most distally from the point of laser application on the 3rd postoperative day. The combined application of red and infrared lasers resulted in the most evident systemic effect on the repair of skin wounds produced in rats. 


\section{Acknowledgments}

This study was supported by Coordenação de Aperfeiçoamento de Pessoal de Nível Superior, Brazil.

\section{References}

1. Belkin, M., Schwartz, M. (1989). New biological phenomena associated with laser radiation. Health Phys. 56, 687-690.

2. Al-Watban, F.A., Zhang, X.Y. (1999). The acceleration of wound healing is not attributed to laser skin transmission. Laser Therapy. 11, 6-10.

3. Mendez, T.M, Pinheiro, A.L., Pacheco, M.T., Nascimento P.M., Ramalho L.M. (2004). Dose and wavelength of laser light have influence on the repair of cutaneous wounds. J. Clin. Laser Med. Surg. 22, 19-25.

4. Pinho de Oliveira, R.A. (2002). Efeitos da fototerapia na cicatrização de feridas cirúrgicas produzidas com o laser de $\mathrm{CO}_{2}$ [thesis]. Porto Alegre (RS): Faculdade de Odontologia da PUCRS.

5. Rochkind, S., Rousso, M., Nissan, M., Villarreal, M., Barr-Nea, L., Rees, D.G. (1989). Systemic effects of low-power laser irradiation on the peripheral and central nervous system, cutaneous wounds, and burns. Lasers Surg. Med. 9, 174-182.

6. Gál, P., Vidinský, B., Toporcer, T., et al. (2006). Histological assessment of the effect of laser irradiation on skin wound healing in rats. Photomed. Laser Surg. 24, 480-488.

7. Basford, J.R., Hallman, H.O., Sheffield, C.G., Mackey, G.L. (1986). Comparison of cold-quartz ultraviolet, low-energy laser and occlusion in wound healing in a swine model. Arch. Phys. Med. Rehabil. 67, 151-154.

8. Saperia, D., Glassberg, E., Lyons, R.F., et al. (1986). Demonstration of elevated type I and type III procollagen mRNA levels in cutaneous wounds treated with helium-neon laser. Proposed mechanism for enhanced wound healing. Biochem. Biophys. Res. Commun. 138, 1123-1128.

9. Goldin, J.R. (1995). Pesquisa em saúde: normas leis e diretrizes. 2nd ed. Porto Alegre: HCPA.

10. Kana, J.S., Hutschenreiter, G., Haina, D., Waidelich, W. (1981). Effect of low-power density laser radiation on healing of open wounds in rats. Arch. Surg. 116, 293-296.

11. Hunter, J., Leonard, L., Wilson, R., Snider, G., Dixon, J. (1984). Effects of low energy laser on wound healing in a porcine model. Lasers Surg. Med. 3, 285-290.

12. Weber, J.B., Pinheiro, A.L., de Oliveira, M.G., Oliveira, F.A., Ramalho, L.M. (2006). Laser therapy improves healing of bone defects submitted to autologous bone graft. Photomed. Laser Surg. 24, 38-44. 
13. Maiya, G.A., Kumar, P., Rao, L. (2005). Effect of low helium-neon (He-Ne) laser irradiation on diabetic wound healing dynamics. Photomed. Laser Surg. 23, 187-190.

14. Reddy, G.K., Stehno-Bittel, L., Enwemeka, C.S. (2001). Laser photostimulation accelerates wound healing in diabetic rats. Wound Repair Regen. 9, 248-255.

15. Rabelo, S.B., Villaverde, A.B., Nicolau, R., Salgado, M.C., Melo Mda, S., Pacheco, M.T. (2006). Comparison between wound healing in induced diabetic and nondiabetic rats after low-level laser therapy. Photomed. Laser Surg. 24, 474-479.

16. Schindl, A., Heinze, G., Schindl. M., Pernerstorfer-Schön, H., Schindl, L. (2002). Systemic effects of low-intensity laser irradiation on skin microcirculation in patients with diabetic microangiopathy. Microvasc. Res. 64, 240-246. 